\title{
Efficiency Problem of renewable energetics systems in the context of «smart house» concept
}

\author{
Ibragim Suleimenov ${ }^{1}$, Zaure Egemberdieva ${ }^{2}$, Akhat Bakirov $^{2, *}$, Saltanat Baipakbayeva ${ }^{3}$, \\ Eldar Kopishev ${ }^{4}$, and Grigoriy Mun ${ }^{5}$ \\ ${ }^{1}$ National Academy of Engineering of the Republic of Kazakhstan, Bogenbai batyr, 80, 050010, \\ Almaty, Republic of Kazakhstan \\ ${ }^{2}$ Almaty University of Power Engineering and Telecommunications, Baytursynov, 126/1, 050013, \\ Almaty, Republic of Kazakhstan \\ ${ }^{3}$ Institute of Information and Computational Technologies, Pushkin, 125, 050010, Almaty, Republic \\ of Kazakhstan \\ ${ }^{4}$ L.N. Gumilyov Eurasian National University, Satbaev, 2, 010008, Nur-Sultan, Republic of \\ Kazakhstan \\ ${ }^{5}$ Al-Farabi Kazakh National University, al-Farabi, 71, 050040, Almaty, Republic of Kazakhstan
}

\begin{abstract}
Considering the question of efficiency of alternative energy systems, producing electricity from the position of interests of households it acquires an unexpected sound. The efficiency can be low (about 7-10\%) providing that the heat released in converting is targeted i.e also used on the needs of households. In such an approach low efficiency becomes acceptable since the most part of energy usage in households is spent on systems using the heat (hot water supply etc.) even in the summer period. It is shown that converting of energy providing targeted heat dissipation can be provided by the phenomenon of generation of electromotive force by flowing of the low-speed, low-molecular salt current trough the polymeric sewed net; relevant experimental results are presented. Basing on the analysis of low-molecular ions currents balance by flowing of solution through the gel, the theory of the phenomenon is proposed. It is shown that the described phenomenon and based on its energy converting systems can be considered as an example illustrating the perspectivity of the new paradigm of green energetics development. Given paradigm is based on principles of technical and humanitarian knowledge convergence and is completely consistent with the conclusion that the environmentfriendly technologies development is a technical and social issue at one time.
\end{abstract}

\section{Introduction}

The question about devices' efficiency providing renewable energy utilization (mainly wind and solar) is generally one of the main for green energetics. It is enough to mention

* Corresponding author: axatmr@mail.ru 
different efforts of various researching groups directed on increasing the efficiency of photoelectric panels [1-3].

At the same time in the current literature often can be met works asserting the thesis proclaiming that the green energetics development is not a technical but social issue [4-7]. This thesis is completely connected with modern ideas of convergence of technical and humanitarian knowledge [8]. Given ideas are transformed in particular into the promotion of green education practices [9-11], green art concept [12], etc.

One of the points of making such efforts is also the smart house concept [13-14], which is considered not only from the point of enhancement of habitat comfort and increasing of the effectiveness of resources usage but also from the point of mass consciousness transformations, targeted to the creation of ecological thinking.

It needs to be emphasized that the basic ecologically-orientated smart house concepts envisage the full energy provision of the individual household received from renewable sources. Thus, the question about its efficiency is absolutely actual for implementation of them into life. This work represents that the social-orientated smart house concept allows considering the question of efficiency of devices, providing the utilization of renewable energy, used by housewives, from a different point of view.

\section{Smart house and the structure of power usage in a household.}

Obviously, when a smart house concept is introduced into the practice, it is advisable to orientate on individual households. Implementation of any innovations requires overcoming the barriers, including psychological ones. Thus, it is complicated to predict the occurrence of different obstacles during the implementation of even separate elements of smart house concept in apartment houses (buildings and locations originally orientated on smart house concept are the exceptions $[15,16]$. However, they are not widespread)

Let us consider the structure of power usage of individual households in the Republic of Kazakhstan in the summer period (table 1). The table based on a direct calculation of energy consumption of 20 Kazakhstan households, related to various social groups in the summer period.

It needs to be emphasized that represented table is related to the summer period, however, it is noticeable that almost the half of energy consumption is connected to the systems that use heating elements (hot water supply, cooking, etc.)

Moreover, the table shows that only a little part of the energy is being used on a supply of systems, for which consumption of energy is crucial (lighting systems, calculating systems and other gadgets). Precisely, all household systems consuming energy can be classified into three groups by nature of use (converting). Systems that are ultimately (considering the efficiency) converting consuming energy into the heat energy (household irons, gas stoves or other stoves, etc.) are related to the first group. Systems, consuming the mechanical energy of rotation of one or another element of construction (work of a refrigerator is provided by rotation of compressor elements, providing the pumping of coolant along the contour of cooling [17]) could be related to the second group. In the third group, there are household systems for which consumption of that or another energy is crucial or the refusal of using exactly this form of energy is connected with the technical difficulties.

It should be noticed that considering the usage of wind energy, in the typical pointing of question, the paradoxical situation is formed relating to the systems of the second group. Efforts of wind systems inventors are spent on converting the energy of mechanical rotation into electricity (it is connected with unproductive looses), further electric energy converts into the energy of rotation of the working construction unit. (also with the efficiency unequal to $100 \%$ ). This thermodynamic nonsense could be eliminated by using the next 
idea: there are plenty of approaches to using the energy of rotation, moreover, this is not necessary to be connected with the usage of mechanical joints.

Table 1. The structure of power usage by Kazakh housewives in the summer period (heating systems are not used).

\begin{tabular}{|c|c|c|}
\hline Type of household energy usage & $\begin{array}{c}\text { Percentage of total } \\
\text { power consumption }\end{array}$ & $\begin{array}{c}\text { Classification } \\
\text { group }\end{array}$ \\
\hline Hot water supply & $45 \%$ & 1 \\
\hline $\begin{array}{c}\text { Heating devices of different } \\
\text { purpose (cooking etc.) }\end{array}$ & $20 \%$ & 1 \\
\hline $\begin{array}{c}\text { Providing of the comfortable } \\
\text { environment (air conditioning, } \\
\text { systems of cleansing) }\end{array}$ & $4 \%$ & 2 \\
\hline $\begin{array}{c}\text { Washing machine, dishwasher } \\
\text { Lighting systems (LED lighting) }\end{array}$ & $13 \%$ & 3 \\
\hline $\begin{array}{c}\text { Refrigeration devices } \\
\text { Computers and TVs, gadgets ( } \\
\text { game consoles, etc.), security } \\
\text { systems. }\end{array}$ & $3 \%$ & 3 \\
\hline $\begin{array}{c}\text { including cell phones, children's } \\
\text { gare }\end{array}$ & & 2 \\
\hline
\end{tabular}

The above facts in combination with ideas of the social character of the majority of green energetics problems allow taking another look to the question of renewable energetics systems efficiency.

\section{Opportunities for practical usage of low-efficiency energy converters}

In terms of thermodynamics, the low efficiency of that or another energy converter means that certain parts of it convert into heat. Considering the efficiency of energy converter abstractedly, this part should be related to unproductive loses. However, if to consider the structure of household energy consumption (Table 1), it is possible to take another look at this question, picture 1 .

Suppose that the energy converter, designed for electricity production is coupled with the element of support of «smart house» providing either room heating or hot water supply (picture 1б). In this case, the efficiency of such a converter could be made low enough, to be precise, so as it is dictated by the structure of household energy consumption, shown in Table 1. It is enough to realize the device with such efficiency, which is determined by given needs, and unproductive losses of energy in this case being converted into the heat will provide the heating. 


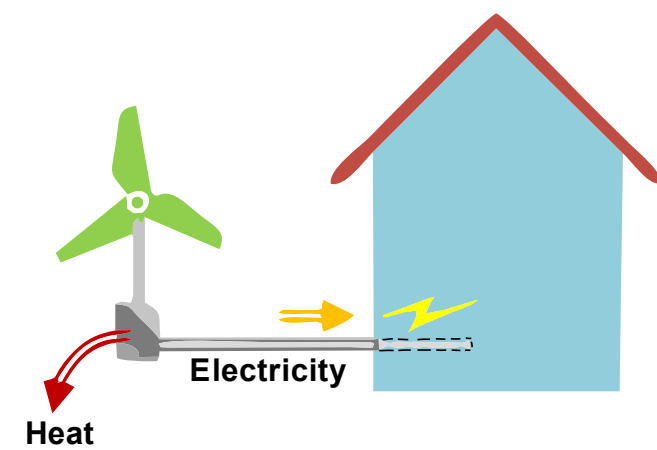

a)

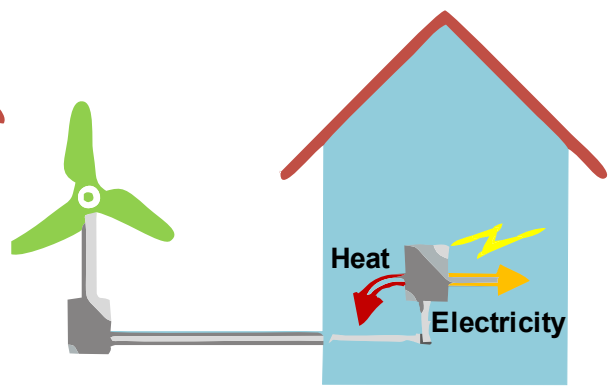

b)

Fig. 1. Illustration of usage of «low efficiency»; a) -traditional approach, b) - approach, based on consideration of «smart house» as an opened thermodynamic system.

As it is demonstrated in table 1, the efficiency of a converter (considering the winter period is applicable to latitudes of Almaty city) with such a campaign could be made extremely low - about $10 \%$. This significantly broadens the physical principles that could be used to get electricity. (It is appropriate to notice that the method based on the coupling of photoelectric panels with heating elements was discussed in scientific literature before [18-19], however, it is not developing systematically.) However, there is a problem - to provide the converter with electricity (with minimal losses), which could be far removed from primary units of the system. The most simplified method of energy transfer is based on the excitement of low-speed liquid flow in closed contour. Show that during the usage of low molecular salt solutions as working liquid this method could be used.

\section{Physico-chemical prerequisites for parallel energy conversion of the low-speed flow of low molecular salts solution into the heat and electricity}

Of the most common electrophoretic phenomenon is the occurrence of flow potential [38], when a low molecular salt solution crosses the send layer potential difference occurs. This potential difference is based on the deformation of double electric layers in the border between send particles and the solution, caused by the movement of liquid. The analogical effect occurs when the low molecular salt solution crosses the polyelectrolyte hydrogel layer. This effect from the point of principle of Onzager [20] is a pair to the effect of creating the possibility for electrodialysis [21]. However, in literature not from experimental nor from scientific points of views it was not considered in attitude to polymeric hydrogels.

The scheme of the experiment, demonstrating the occurrence of potential flow analogue is shown in picture 2. It includes:

- A tube filled with hydrogel (1);

- Membranes (2) and (3), separating the active substance from low molecular salt solution;

- Volume (4), filled with a low molecular salt solution,

- Buffer volume (5), serving for deletion of low molecular salt crossed the hydrogel layer;

- Electrodes (6), serving for measurement of dynamic potential difference;

- Precise voltmeter (7), providing the opportunity of conducting the measurement up to the millivolt units. 


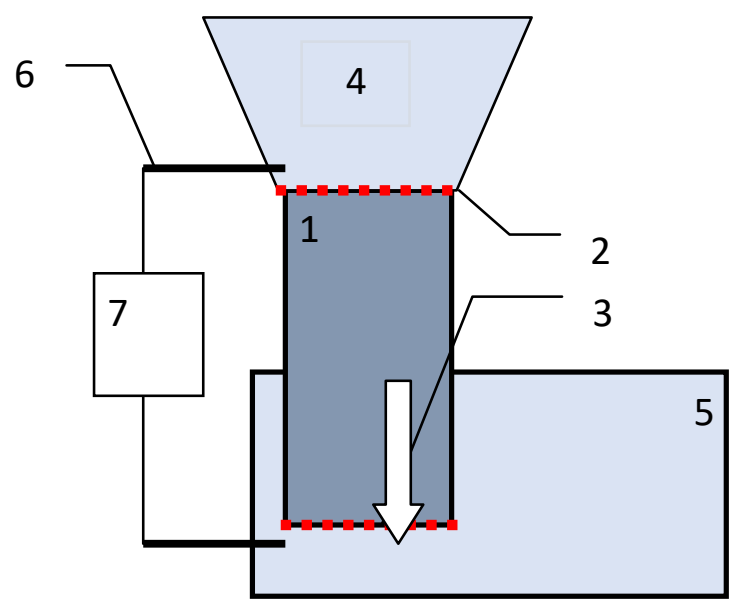

Fig. 2. Scheme of experiments of detection the potential of solution flow across the gel.

During experiments gel (1) was placed between membranes (2) and (3). The volume (4) above the gel was filled with a low molecular salt solution, where lower buffer volume (5) initially was filled with whether deionized water or with the low molecular salt solution of the same concentration as the volume. (5) The difference of electric potentials was registered during the flow of the salt solution across the gel; In parallel, a change in altitude of solution column above the gel and the potential difference as the function of time was measured.

The volume filled with buffer under the gel based on polyacrylic acid (industrially made hydrogel was used) was $300 \mathrm{ml}$. Glass cylinder $20 \mathrm{~mm}$ in diameter filled with a turgid PAA gel of $50 \mathrm{~mm}$ high was used. The initial altitude of $0,05 \mathrm{M} \mathrm{KCl}$ solution above the gel was also $50 \mathrm{~mm}$. An example of the experimental measurement results is shown in picture 3 .

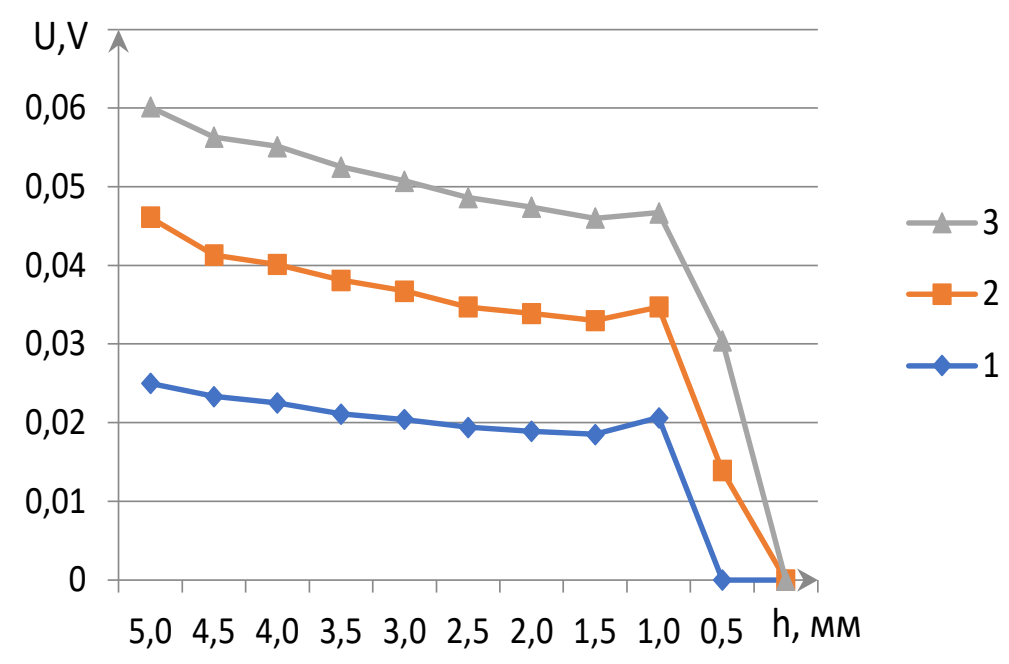

Fig. 3. Dependences of obtained values of difference of potentials above and under the hydrogel from the altitude of solution column above the gel filling the buffer volume with the solution of the same concentration as above the gel for 3 different lengths of hydrogel column; 25(1), 40(2), 50(3) mm. 
It can be seen that during the crossing of low molecular salt through the hydrogel column, the difference of potentials (EMF) occurs. This EMF could be used for the realization of heating elements and forgetting the electricity. To show that, let's consider the occurrence of the difference of potentials mechanism, detected in experiments.

\section{The mechanism of flow potential analog occurrence}

Let's consider the gel sample (to simplify the calculations without loss of generality, it can be considered a homogeneous material with a spatial distribution density of functional groups), through which the 1:1 low molecular salt solution flow. The fact of flowing of solution through the gel material means that beyond the gel volume the nonzero flows of low molecular ions which's direction is the same (in contrast to the situation when the ions move under the electricity influence) are existing. Let's consider the case when the mobilities of $b$ cations and anions of 1:1 salt is the same, where noted values stay constant beyond and inside the hydrogel (such assumption responses the case that the gel has a high degree of swelling).

Then, the values of positive and negative ions in solution beyond the gel (index 1 corresponds to this sphere) could be noted as

$$
\begin{aligned}
& j^{+}=v_{0} n_{1}^{+} \\
& j^{-}=v_{0} n_{1}^{-}
\end{aligned}
$$

The equations (1) and (2) express the fact that low molecular salt ions are carried with the liquid. (However, the concentration of ions of both signs inside the polyelectrolyte gel substantially differs from its concentration outside (The redistribution of concentrations takes place [22-25]) i.e functions of the concentration between gel and solution experience the leap. At the same time, functions that describe the spatial distribution of currents on the boundary must be continuous, otherwise an accumulating of charge would be taking place on this boundary. (Such accumulating actually happens but only before the stationary condition) Since the speed of the liquid flow on the considered boundary is also continuous function, boundary conditions consisting of function continuity can not be done if not to consider extra factors. It is becoming obvious when equations (1) and (2) are demonstrated considering the difference in concentrations of the mobile ions outside and inside of hydrogel.

$$
\begin{aligned}
& j^{+}=v_{0} n_{2}^{+} \\
& j^{-}=v_{0} n_{2}^{-}
\end{aligned}
$$

For the case of the cell, carrying the negative charge inequalities are fulfilled.

$$
n_{2}^{+}>n_{1}^{+}=n_{1}^{-}, n_{2}^{-}<n_{1}^{+}=n_{1}^{-}
$$

Which are determined by the presence of own ions forming during the dissociation of the polyelectrolyte cell substance and by the effect of redistribution effect. Boundary conditions are met if to suppose that inside the area 2, filled with hydrogel voluntary occurs the electric field, which inhibits the movement of the charged particles with higher concentration and accelerates them with lower concentration. Then, instead of ratios (3) and (4) should be written

$$
j^{+}=v_{0} n_{2}^{+}+b E n_{2}^{+}
$$




$$
j^{-}=v_{0} n_{2}^{-}-b E n_{2}^{-}
$$

the determined value of electric field tension, therefore the considered approximation expressed by the formula

$$
E=\frac{v_{0}\left(n_{2}^{-}-n_{2}^{+}\right)}{b\left(n_{2}^{+}+n_{2}^{-}\right)}
$$

From the obtained expression (8) it has seen that the intensity of the electric field completely determined by the difference between the concentration of positive and negative mobile ions inside the hydrogel. This result also shows that the occurrence of flow potential analog is due to the difference of concentrations between positive and negative ions outside and inside the gel. This mechanism could be a base of heat converter responding to criteria of applicability for «smart house», considered above.

\section{Device for parallel converting of the energy of the low-speed flow of the low molecular salts solution into the heat and electricity}

Following device providing the converting of the energy of law molecular salt solution circulation into the heat (the most obvious way of providing the circulation of the liquid in closed contour is wind energy, but for this effect, solar energy could be also used [26]) could be considered.

It is significant that considered below construction allows making the heat releasing locally. This allows suggesting that a given system provides the converting of the energy into the heat to create the heating points of a smart house.

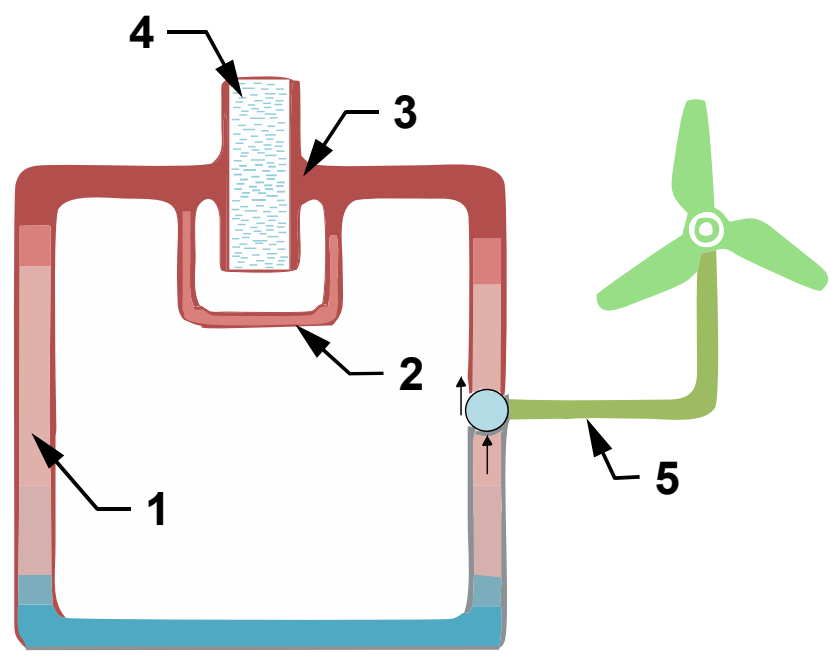

Fig. 4. Scheme of heat release during the circulation of a solution in closed contour.

Device (fig.4) includes circulation contour 1, containing heat-releasing points 2 and 3 , based on specific properties of sewed polymeric nets. Circulating inside the contour liquid expressed by low molecular salt solution crosses points 2 and 3, which provide the heat release at the local point of the contour. Heat releasing is provided by physical and chemical phenomenon considered above. Into the point 3 , expressed by extended tube, plate out of sewed electrolyte net 4 is being inserted. The extended cross-sectional area of the 
tube is made for decreasing the hydrodynamic resistance, created by the net. Parallel to the given point, tube 2 is included relative to the small cross-section.

The tube is only filled with the solution, its small cross-section is chosen according to the condition when hydrostatic resistance of points 2 and 3 is about the same. Therefore, part of the solution circulating in contour 1 is leaking out of the plate 4. As it is shown above leaking of the solution trough the plate is accompanied by the occurrence of a difference of potentials on the end edge of the plate.

The arising difference of potentials leads to the occurrence of nonzero electric currents inside the inserted contour, formed by the elements (2) and (3). Wherein the Om resistance of the point (3) is much less than the resistance of tube (2) because of the significant difference in cross-sections. Given contour has significant Om resistance, the value of which depends on the concentration of working solution and the cross-section of the tube (2). Therefore, inside the given element contour Joule heat releases. In other words, the wind energy, transmitted across the point (5) to the liquid, circulating in the contour is locally converted into the heat. Therefore, such systems could be used for heating of residential buildings using wind energy.

The system demonstrated in picture 4 is also able to produce electric energy at the same time. To be more precise, this system is a converter of liquid circulation energy into the electricity, however, it has low efficiency. However, it is not an obstacle for household usage. Moreover, exactly the low efficiency could be converted into the advantage of this system, since the main part of produced energy is converted into the heat, that is the main purpose of the system. Electricity produced as a certain addition.

\section{Conclusion}

Thus, if to consider the interests of specific households, the question of efficiency of systems, providing the converting of solar and wind energy into the electricity, appears in a different form. The structure of energy consumption by real household makes sense that there is no need to increase the energy more than $10 \%$ in case of the rest $90 \%$ de-facto converted into the heat are used targeted for the provision of households by the heat. (hot water supply, cooking, etc.)

Systems where «heat losses» are utilized targeted could be realized by the simple approach, in particular, using the electrophoretic phenomenon.

It should be noticed that in this work only one example of such converting of energy was considered. Unique properties of polyelectrolyte hydrogels, which were investigated for by Kazakh physical-chemical school a long time allows to realize the whole spectrum of such systems, where the suggested approach is being realized. The approach taking the real energy consumption structurer into account could be realized in interests of not only households but also in interests of small and medium agribusiness. In other words, the suggested approach creates bases of development of the principally new paradigm of green energetics development de-facto based on principles of natural-scientific, technical and humanitarian knowledge convergence. Green energetics is a sphere where given principle appears in all completeness, which is confirmed by the results of this work. The development of green energetics (creation of green economics, nature-friendly economics) can be successful only when it considers technical and social factors. 


\section{References}

1. H. Lu, L.-Z. Zhang Renew. ENERGY 135 21-31 (2019) DOI: 10.1016/j.renene.2018.11.096

2. A. Jha. P.P. Tripathy Renew. ENERGY $135856-865$ (2019) DOI: https://doi.org/10.1016/j.renene.2018.12.032

3. A.M. Freitas, R.A.M. Gomes, R.A.M. Ferreira, M.P. Porto Renew. ENERGY $1351004-$ 1012 (2019) DOI: 10.1016/j.renene.2018.12.051

4. M. Wolsink, Renew. Sustain. ENERGY $16 \quad 822-835$ (2012) DOI: https://doi.org/10.1016/j.rser.2011.09.006

5. D.-X. Zhao, B.-J. He, C. Johnson, B. Mou Renew. Sustain. ENERGY 51 1594-1609 (2015) DOI: https://doi.org/10.1016/j.rser.2015.07.072

6. D. Bechtsis, N. Tsolakis, D. Vlachos, E. Iakovou J. Clean. Prod 142 3970-3984 (2017) DOI: https://doi.org/10.1016/j.jclepro.2016.10.057

7. A. Mardani, E.K. Zavadskas, Z. Khalifah, N. Zakuan, A. Jusoh, K.M. Nor, M. Khoshnoudi Renew. Sustain. ENERGY 71 216-256 (2017) DOI: https://doi.org/10.1016/j.rser.2016.12.053

8. S. Uruena Lopez Filos Philosophy of science: A discipline between the scientific and humanistic cultures. Themata-revista 113-132 (2018)

9. M. Karpudewan, Z. Ismail, W.-M. Roth Chem. Educ. Res. Pract 13 120-127 (2012) DOI: https://doi.org/10.1039/C1RP90066H

10. M.K. Juntunen, M.K. Aksela Chem. Educ. Res. Pract 15 488-500 (2014) DOI: https://doi.org/10.1039/C4RP00128A

11. E. Nyberg, D. Sanders J. Biol. Educ 48 142-153 (2014) DOI: https://doi.org/10.1080/00219266.2013.849282

12. N. Tubbs Educ. Philos. THEORY 50 1068-1074 (2018) DOI: $10.1080 / 00131857.2017 .1340148$

13. M. Chan, E. Campo, D. Esteve, J.-Y. Fourniols Maturitas $6490-97$ (2009) DOI: https://doi.org/10.1016/j.maturitas.2009.07.014

14. B. Zhou, W. Li, K.W. Chan, Y. Cao, Y. Kuang, X. Liu, X. Wang Renew. Sustain. ENERGY Rev 61 30-40 (2016) DOI: https://doi.org/10.1016/j.rser.2016.03.047

15. M. Somayya, R. Ramaswamy Proceedings of the SUSTAINABLE CITY XI; Galiano Garrigos, $A$ and Brebbia Amsterdam Smart City (ASC): fishing village to sustainable city 204 831-842 (2016) DOI: 10.2495/SC160681

16. M.A.G. Timmer, K. de Blok, T.H. van der Meer J. Acoust. Soc. Am 143 841-857 (2018) DOI: https://doi.org/10.1121/1.5023395

17. S. Choi, U. Han, H. Cho, H. Lee Appl. Therm. Eng. 132 560-574 (2018) DOI: https://doi.org/10.1016/j.applthermaleng.2017.12.133

18. N. Le Pierres, M. Comer, L. Luo, G. Fraisse Int. J. ENERGY Res. 32 1316-1328 (2008) DOI: https://doi.org/10.1002/er.1439

19. N. Arcuri, F. Reda, M. De Simone Sol. ENERGY 105 147-156 (2014) DOI: https://doi.org/10.1016/j.solener.2014.03.034

20. L. Onsager I. Phys. Rev. 37 405-426 (1931) DOI: https://doi.org/10.1103/PhysRev.37.405 
21. J. Garrido Desalination 265 274-278 (2011) DOI: https://doi.org/10.1016/j.desal.2010.07.032

22. T. Budtova, N. Belnikevich, I. Suleimenov, S.Y. Frenkel Polymer (Guildf) 345154 5156 (1993) DOI: https://doi.org/10.1016/0032-3861(93)90262-9

23. T. V. Budtova, I.E. Suleimenov, D.A. Bichutskii, S.Y. Frenkel Vysokomol. Soedin. Seriya a Seriya B 37 1019-1023 (1995)

24. I.E. Suleimenov, G.A. Mun, I.T. Pak, S.B. Kabdushev, Z.A. Kenessova, E.E. Kopishev News Natl. Acad. Sci. Repub. Kazakhstan-series Geol. Tech. Sci. 423 198-205 (2017)

25. T. V. Budtova, I.E. Suleimenov, S.Y. Frenkel Vysokomol. Soedin. Seriya A Seriya B 37 147-153 (1995)

26. M. Mohanraj, Y. Belyayev, S. Jayaraj, A. Kaltayev Renew. Sustain. Energy Rev. 83 124-155 (2018) DOI: https://doi.org/10.1016/j.rser.2017.08.086 